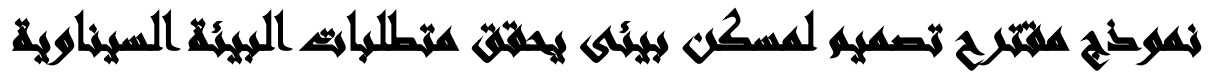

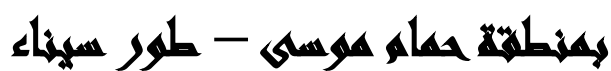

$[1 \cdot]$

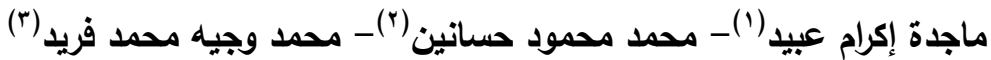

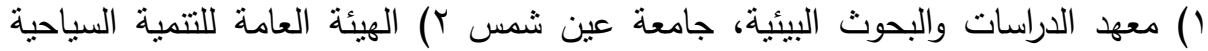
r) حمام موسى، طور سيناء، جنوب سيناء

\section{varill}

تعانى المساكن فى سيناء عامة وفى مناطق توطين البدو خاصة مثال ذللك منطقة حمام

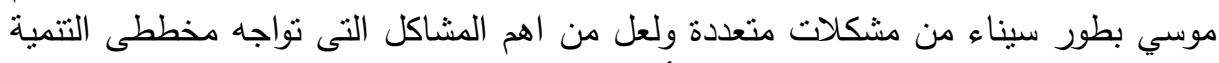

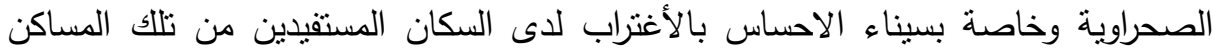

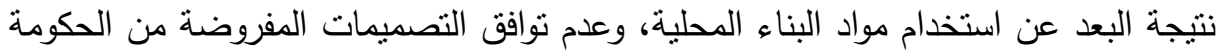

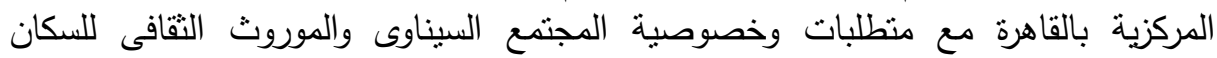

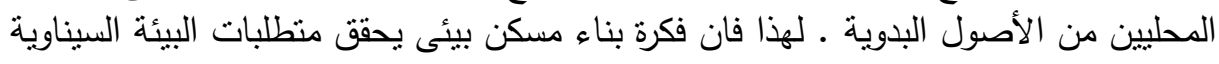

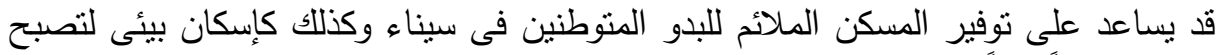

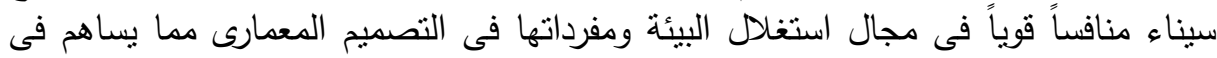

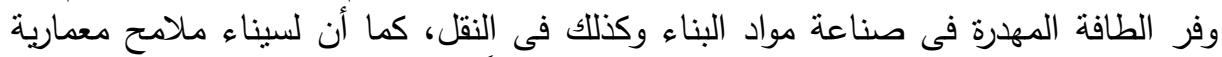
وعمرانية مختلفة، وكذلك البيئة السيناوية تقرض شرورة الئاء

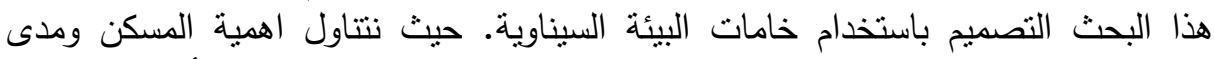

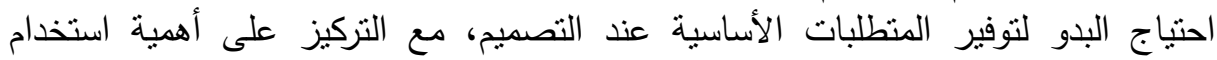

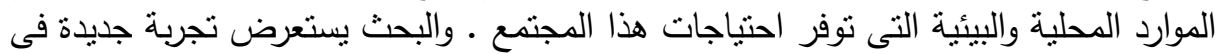
توفير مسكن يحقق متطلبات البدو من التصميم والاستفادة بالتكنولوجيا الحديثة فى توفير وترشيد الطاقة داخل المبنى السكنى.

\section{2and}

هجرة البدو للمساكن وعدم رضاهم عن السكنى فى الاماكن البديلة المشيدة بالخرسانة المسلحة، لعدم توافقها مع منطلبات البيئة السيناوية ذات الخصوصية والطابع المنقرد.

$$
\text { المجلد الخامس والثلاثون، الجزء الثاني، سبتمبر } 7 \text { ا ـ ب }
$$


مجلة العلوم البيئية

معهد الدراسات والبحوث البيئية - جامعة عين شمس

عدم الاستفادة بالموارد المحليةفى عملية البناء خاصة ان سيناء تحتوى على الاحجار الرسوبية والنارية ، كذلك العديد من انواع الرمال والرخام والجرانيت والزلط وخام الاسمنت

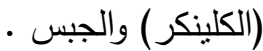

\section{المصني}

- اعداد مقترح لمسكن بيئى يراعى المناخ واحتياجات السكان - - مسكن يستخدم التقنيات الحديثة فى ترشيد الطاقة - - يستخدم مواد بناء محلية من البيئة السيناوية

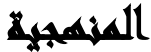

سوف يتبع البحث المنهج الاستتباطى فى الجزء الاول يهتم بدراسة الكليات ويفسر

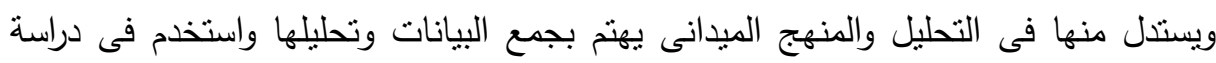

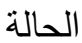

\section{سبريم الختيار اللحاله}

حمام موسى شكل رقم (1) منطقة شديدة التميزعلى ارض سيناء حيث تتمتع المنطقة بمقومات طبيعية فريدة وتستخدم المياه لحمام موسى كعلاج للعديد من الامراض شكل رقم (r) 


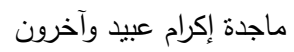

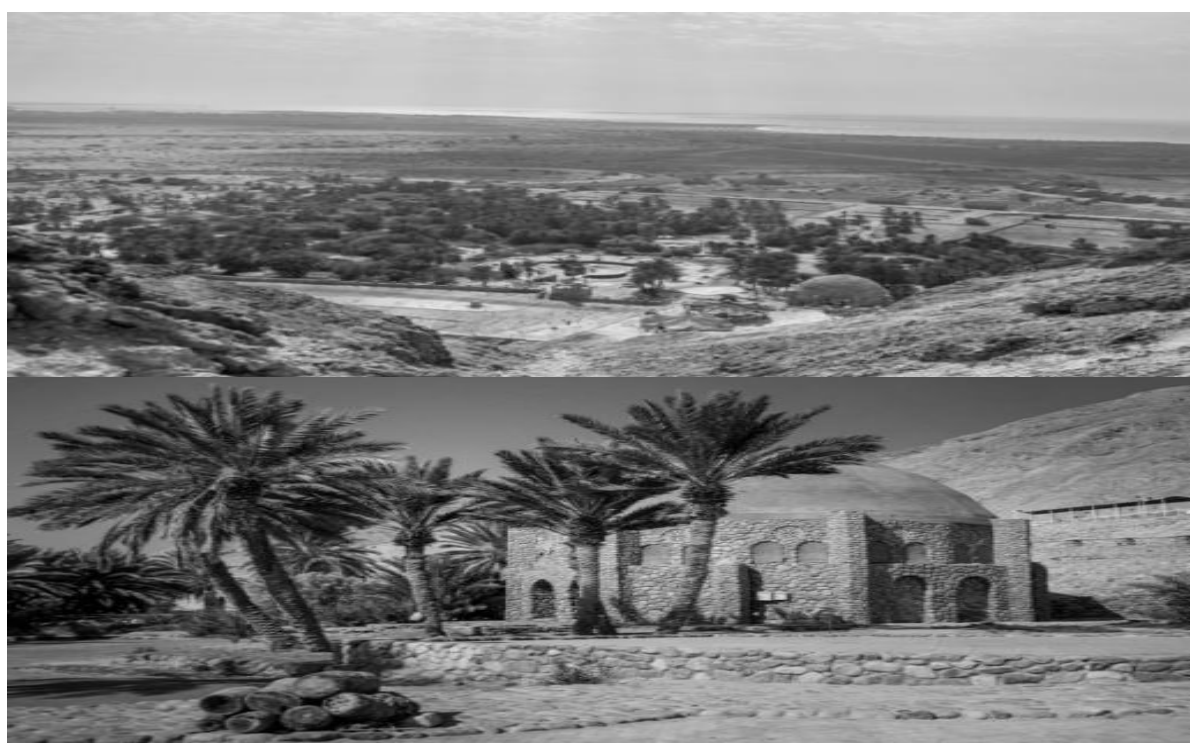

شكل رقم(1): حمام موسى (منطقة الدراسة)

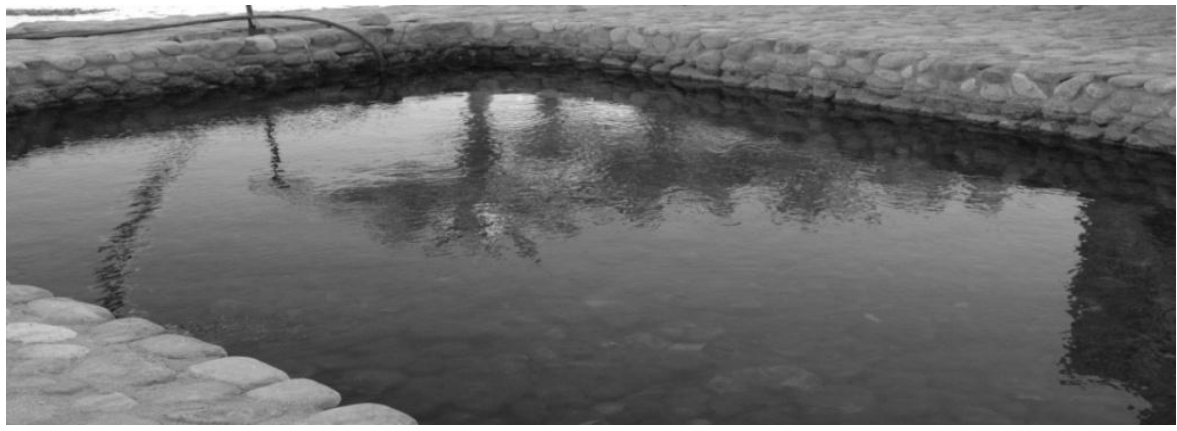

شكل رقم(ץ) : الاستخدام العلاجى لمياه عين حمام موسى.

وصف منطقة الدراسة: تقع منطقة حمام موسى بمدينة طور سيناء على الطريق الرئيسي إلى مدينة شرم الثيخ حيث يحد المنطقة من الغرب كورنيش ساحل خليج السويس، كما يتصل الموقع مباشرة بمداخل المطار الجديد من الناحية الجنوبية، وهو عبارة عن مثلث حدة

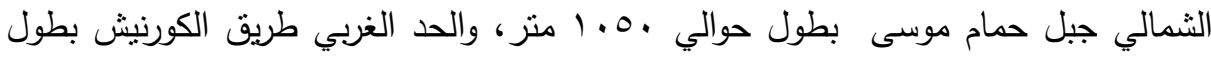

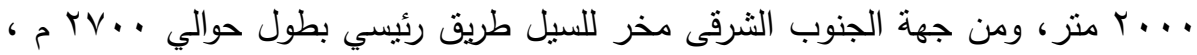

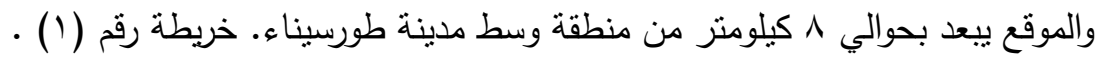

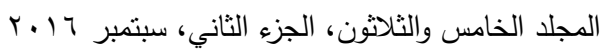




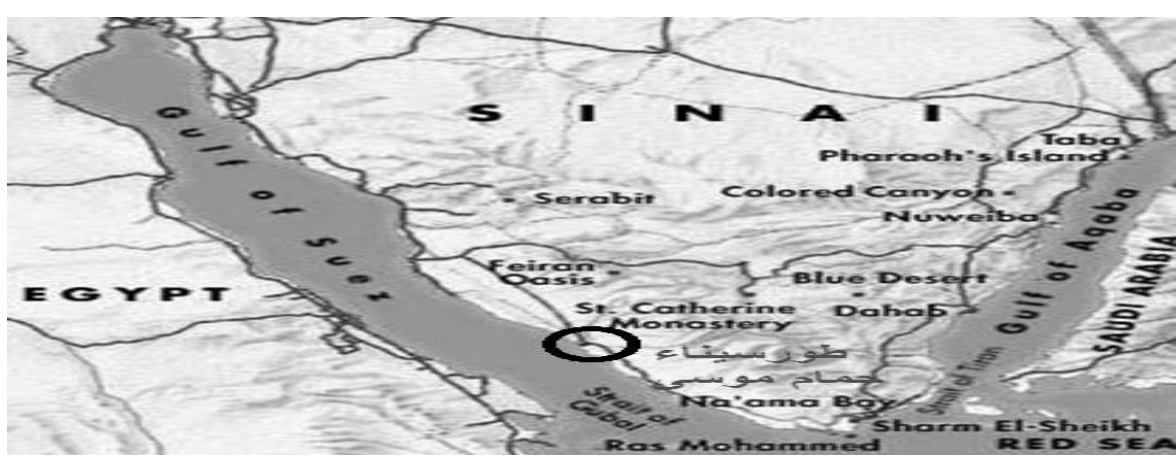

$$
\text { خريطة رقم (1): توضح موقع حمام موسى التهي }
$$

مكونات التصميم: يتكون المسكن الذى يفضله البدوى فى معيشته من المقعد (الديوان) والحوش الواسع وغرف النوم (غرفنتين او ثلاث) والمطبخ والحمام. النموذج المفترح لتصميم المسكن البيئى - محددات التصميم - يعتمد على :

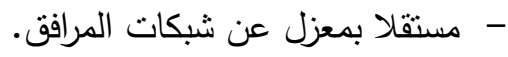

- استخدام الخامات البيئية المتوافرة بالمنطقة شكل رقم( r ). - يتوافق ويتماشى مع طبيعة السكان من البدو واستخداماتهم اليومية. - يراعى الخصوصية كما يراعى الاستقلالية وامكانية التوسع الافقى والرأسى مستقبلاً. - يستفيد من أشعة الثمس وهواء الرياح ، فى الإضاءة والتهوية وتوليد الطاقة. - يتمتع بأقل معدل ممكن من النفاذ الحرارى.

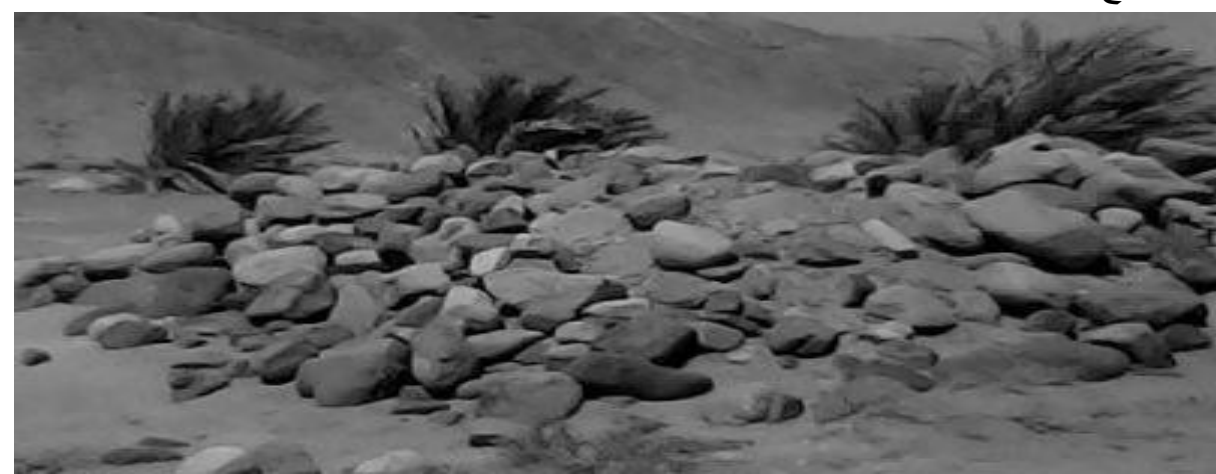

شكل رقم(r): الخامات الطبيعية المستخدمة فى البناء 
هذا ولقد تم مراعاة معظم المعالجات الهندية النى توفر الطاقة وستخدام مواد البناء

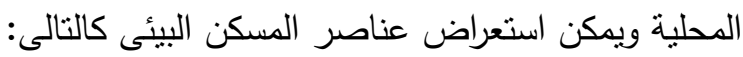
أولاً: مواد البناء المحلية: نم استخدام الدبش فى العمليات الإنشائية لبناء الحوائط

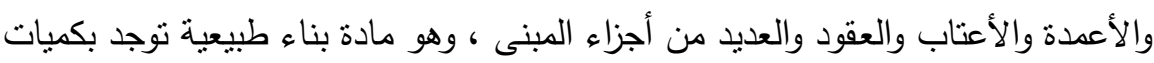
كبيرة فى محاجر كثيرة مثل تلال عيون موسى ، ومحجر الدويقة ، ومحجرة الجامون بحلوان.

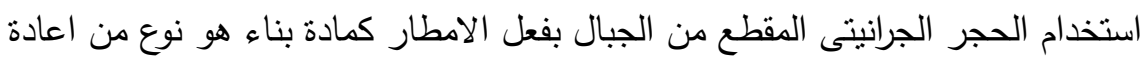

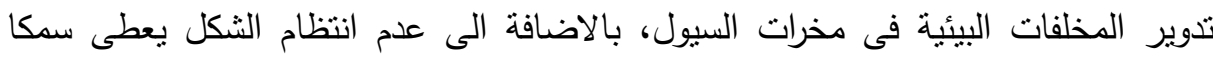
للحائط وتراكيبا هندسية مختلفة لاتحتاج لبياض خارجى، كما يتمتع الحجر الجرانيتى بدرجة

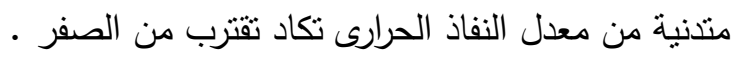
مجروش القشريات (الأصداف) البحرية مع رمل الزجاج الأبيض داخل مونة البناء لنقليل

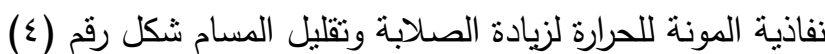

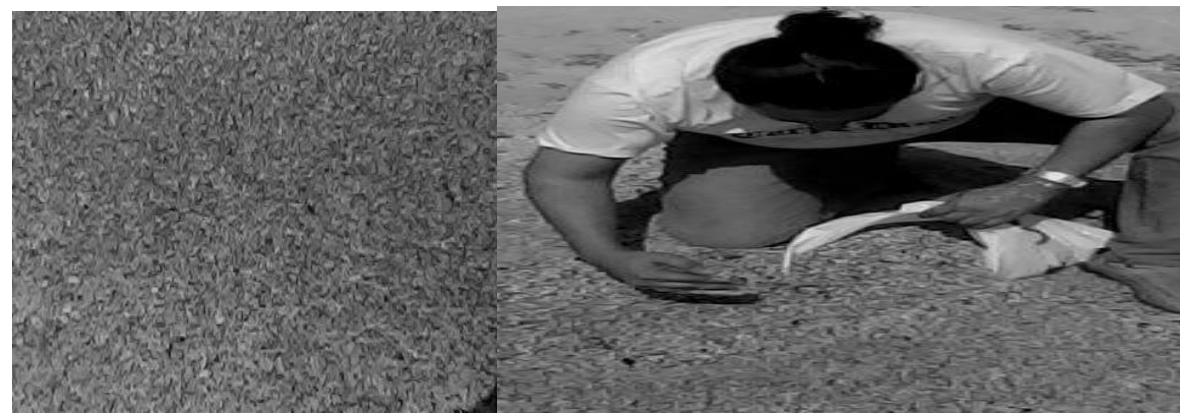

$$
\text { شكل رقم(؟ ): الباحث يجمع القشريات البحرية }
$$

الرمال البيضاء توجد في جبل المنشرح بالحسنة ووادي فللي وجبل يلق وجبل الحلال وجبل منظور ويستخدم في صناعة الزجاج والبللور والسيراميك والسليكون النقي والموصلات

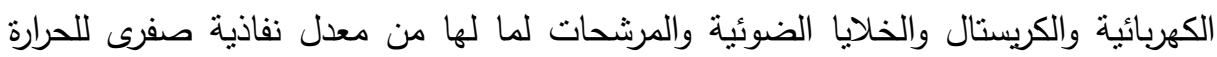

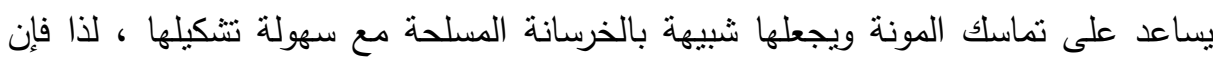

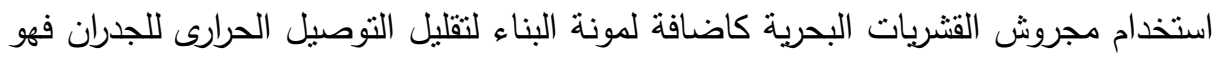
وسيلة فعالة لأستخدام جيد لهذا الكم الضخم من القشريات التى يلفظها البحر على الثواطئ بشكل طبيعى كنوع من اعادة تدوير المخلفات البحرية.

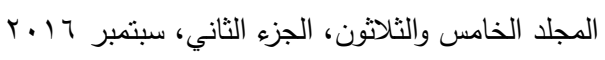


استخدام رمل الزجاج الأبيض الغراء وخليط من المعجون والبلاستيك للطلاء الداخلى.

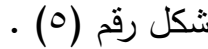

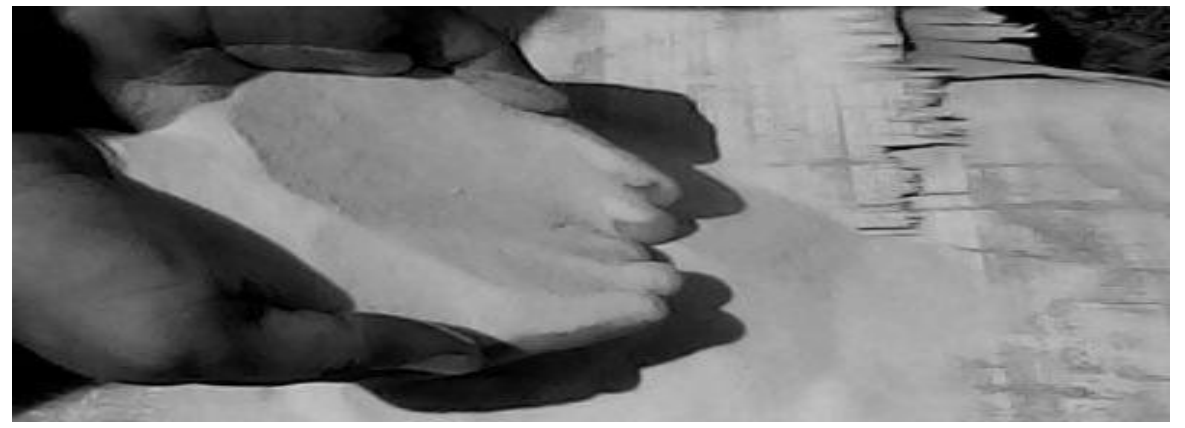

شكل رقم(ه): رمل الزجاج الأبيض شديد النقاء

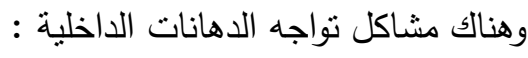

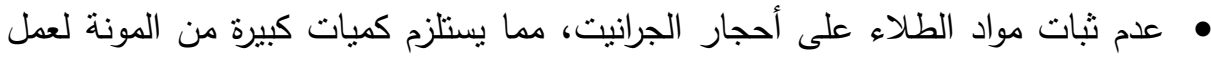

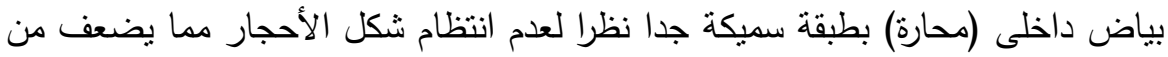

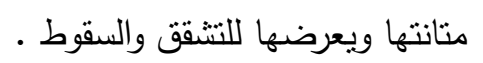
• رطوبة الهواء نظرا لتعرض الواجهات للبحر والذى يمثل العنصر الأساسى للتهوية الطبيعيبة مما يؤثز على كفاءة الطلاء بالمواد التقليدية . وقد أقرت التجارب الميدانية أن استخدام رمل الزجاج الأبيض والغراء وخليط من باءله المعجون والبلاستيك للطلاء الداخلى يكون خليطا متجانسا يسنطيع الالتحام بالأحجار

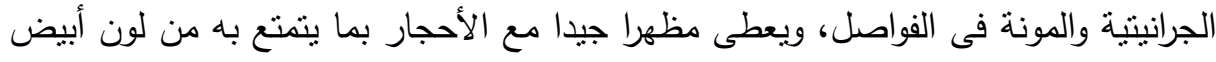

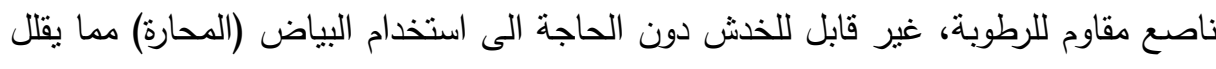

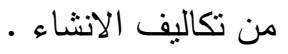


استخدام سقف مركب من الخشب او جذوع النخيل مع ألواح البولى الكربونايت للأسقف

للسماح بالأضاءة الطبيعية نهارا شكل رقم (†) .

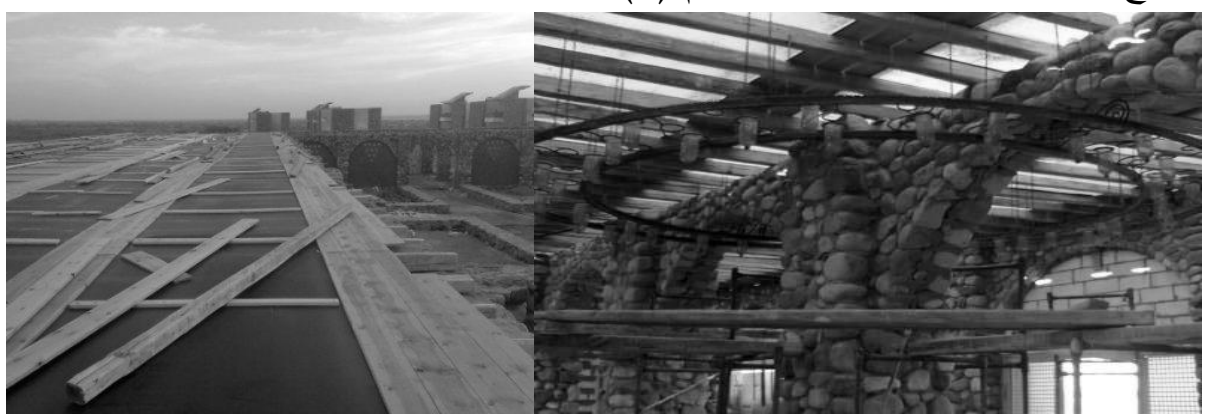

شكل رقم(؟): الاضاءة الطبيعية من خلال استخدام السقف الخشبى مع الألواح

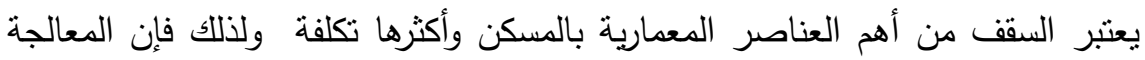

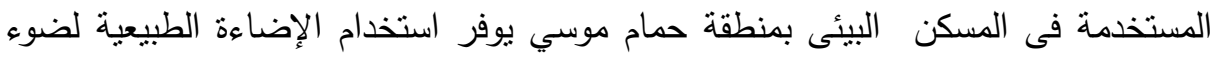

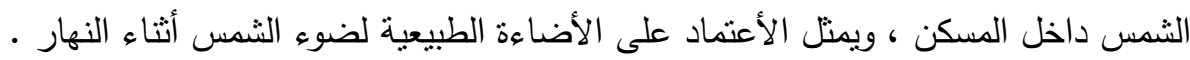

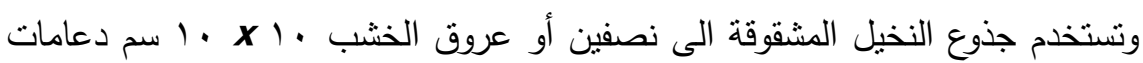

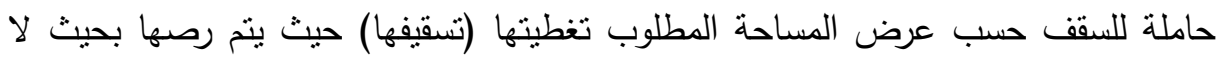
تزيد المسافة البينية عن •ـ سم ثم نغطيتها بالواح خثبية عرض 10 سم بحيث يتم رص بـ ألواح بجوار بعضها من الخشب، يتلوها نصف لوح من البولى كربونايت مقسوم طوليا بحيث

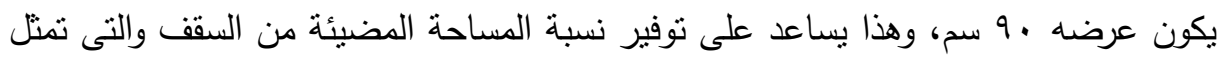

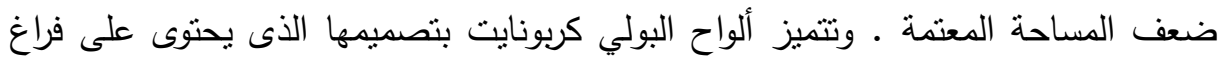

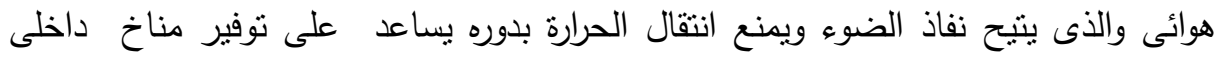
مناسب، ويرشد من استهلاك الطاقة ويساعد على الإعتماد على التهوية الطبيعية داخل فراغ

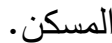


استخدام ملاقف الهواء لعمل تيارات هوائية باردة داخل الغرف. شكل رقم(V)

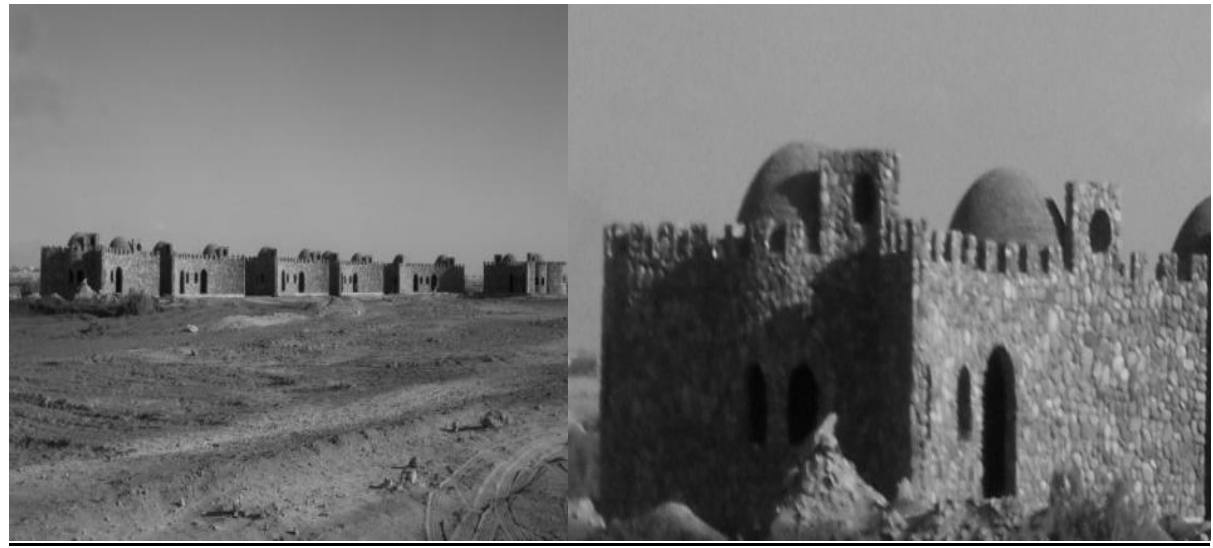

شكل رقم (V): ملاقف الهواء فى المساكن المقترحة

حيث تستخدم ملاقف الهواء من خلال ابراج متوسطة الارتفاع، يزيد ارتفاعها مترين

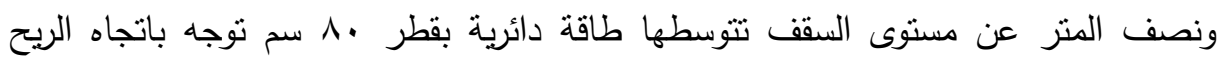

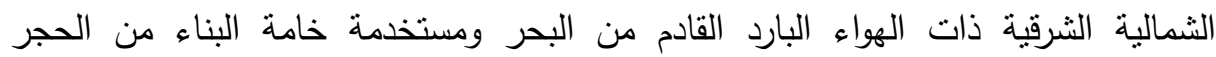
الجرانيتى الخام.

\section{ثانياً: موقد شمسى بالحوش للطهى وسخان شمسى لتسخين المياه:}

الطهي بالطاقة الثمسية يتم عن طريق فرن شمسي وهو جهاز يستخدم أشعة الثمس ملثس

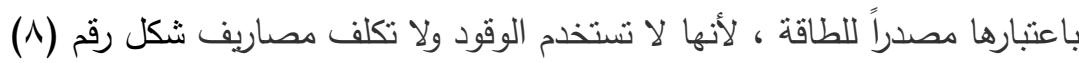


ماجدة إكرام عبيد وآخرون

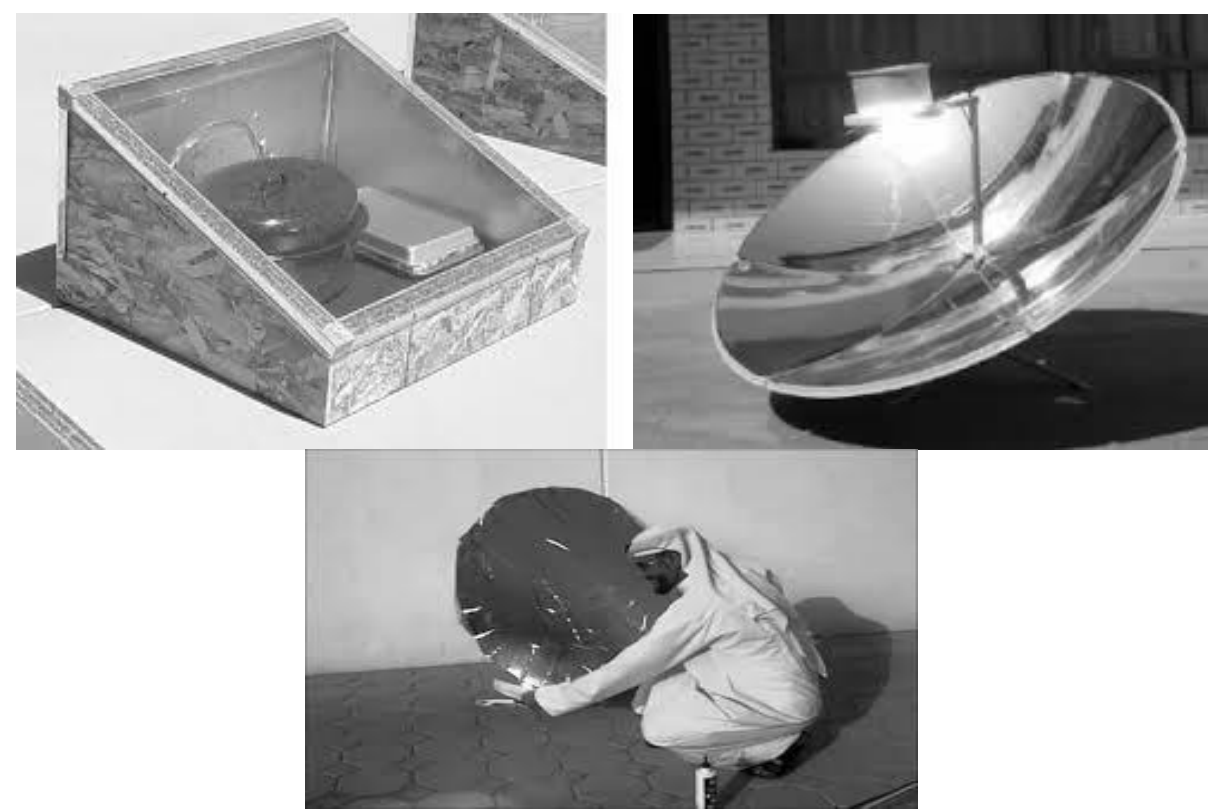

شكل رقم(^): الموقد الثمسى

ويعتمد الطهي بالطاقة الثمسية أو الطباخ الثمسي ، على مبدأ استخدام أشعة الثمس

بشكل مباشر ، والتي يتم عكسها بواسطة مرآة مسطحة خارجية ، لتوجه داخل هذا الفرن البسبط

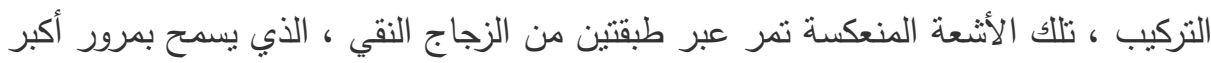
قدر من الأشعة داخل الفرن ، كما يمنعان انعكاس الحرارة من داخل الفرن. ثالثاً: تسخين المياه للاستخدامات اليومية باستخدام الطاقة الشمسية

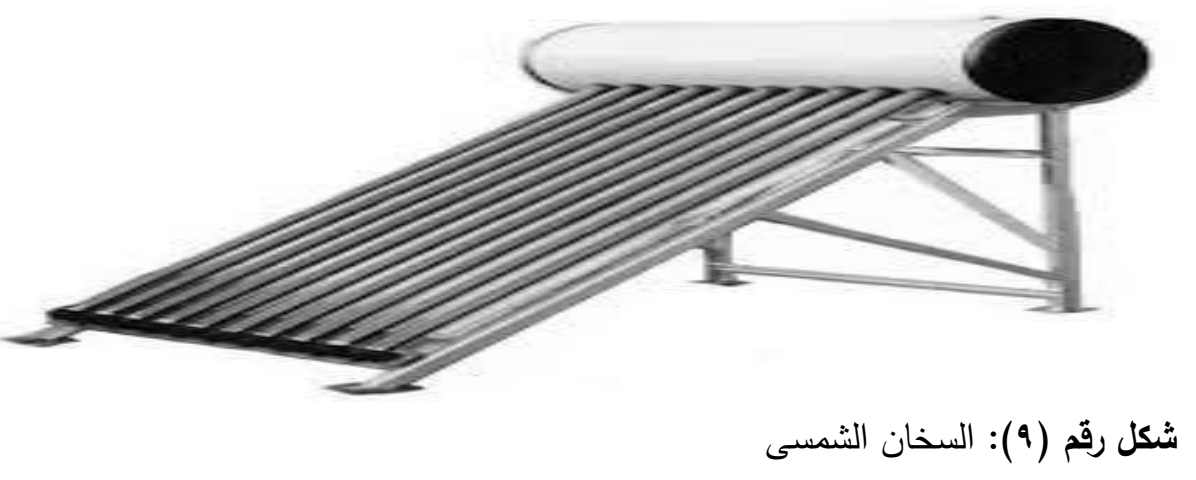

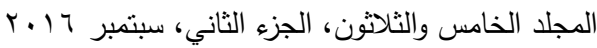


استخدام الألواح الثمسية لنسخين المياه، ويعد نظام الألواح الثمسية لنسخين المياه قادراً على تأمين الثلث من المعدل الطبيعي لأفراد الأسرة من إنتاجها السنوي من إمدادات المادئ المباه

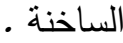

ويمكن الاعتماد على ألواح الطاقة الثمسية لتسخين المياه، بالأضافة إلى ذلك الاعتماد

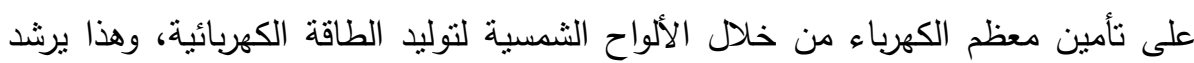
الكثير من الاحتياجات اليومية من الطاقة . رابعاً: إستخدام البيوجاز للإستفادة من المخلقات لترشيد الطاقة التقليدية تتكون وحدة البيوجاز من أربعة أجزاء رئيسية:

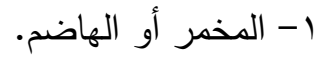

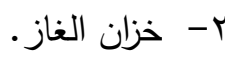
ץ- حوض التغذية بالروث والمواد الخام (حوض الدخول). ع-حوض خروج السماد العضوي (حوض الخروج ).

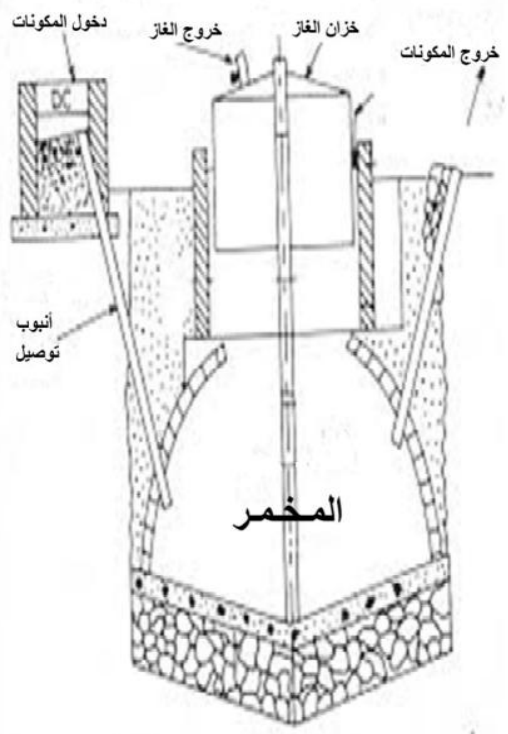

شكل رقم(· ( ): الأقسام الداخلية لوحدة البيوجاز المجلد الخامس والثلاثون، الجزء الثاني، سبتمبر 7 ب ب 
خامساً: استخدام الطاقة الجديدة والمتجددة الطاقة الثمسية

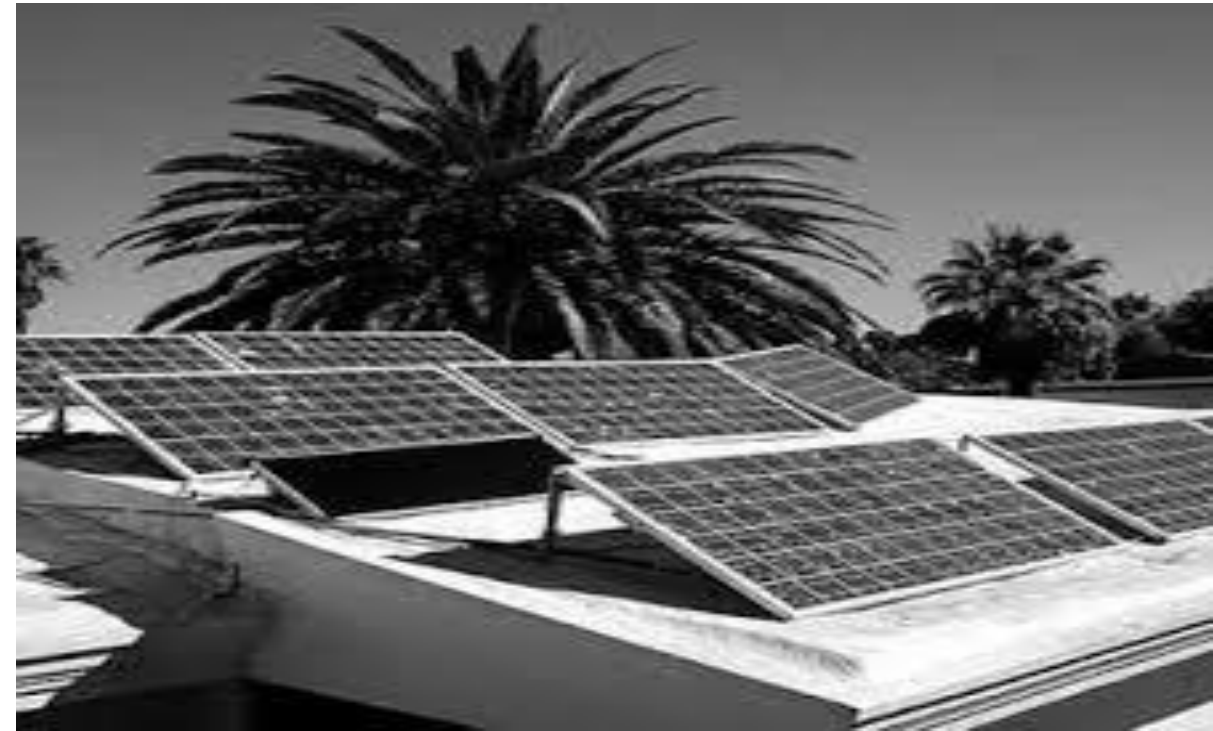

شكل رقم(1 1 ): الألواح الثمسية اعلى سطح النموذج التطبيقى

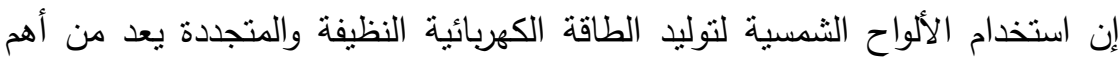
الطرق لتوليد الطاقة الطبيعية من إنتاج منزلي بشكل منعزل عن الثبكة المحلية.

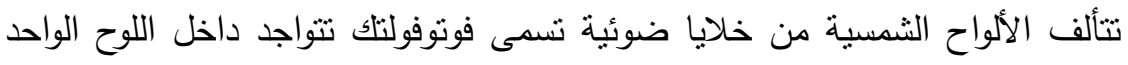
على شكل مصفوفة ذات بعدين • هناك العديد من مزايا استخدام مصفوفة الخلايا الثمسية،

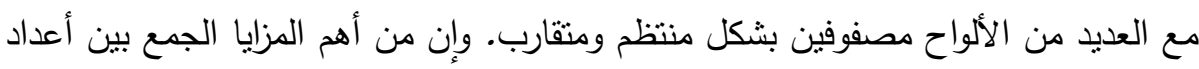
مختلفة من الخلايا لتوفير قدر أكبر من الإنتاج الكهربائي.

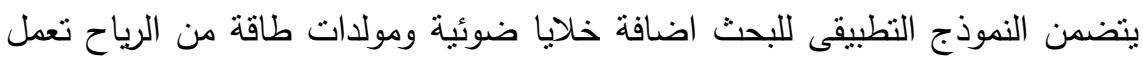
فى مجموعها على توفير احتياجات المسكن من الطاقة مما يجعله نموذجا للمساكن ذاتية

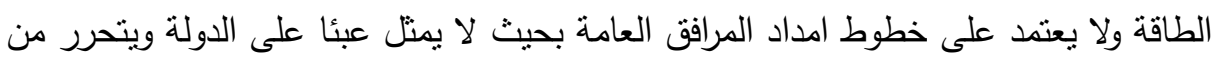
محدودية أماكن انثاؤه بحيث يمكن وبثكل اقتصادى انشاء تجمعات سكنية صغيرة فى أماكن بعيدة عن شبكات المرافق.

$$
\text { المجلد الخامس والثلاثتون، الجزء الثاني، سبتمبر } 1 \text { ا ـ ب }
$$


طاقة الرياح : استخدام طاقه الرياح من خلال تحويل حركة الرياح إلى شكل آخر من أثكال

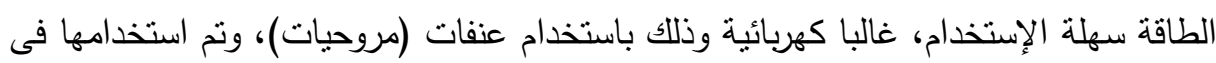
رفع وتحلية المياه من البئر للاستخدامات اليومية للمسكن ولتتغيل السلسبيل (مدرج المياه
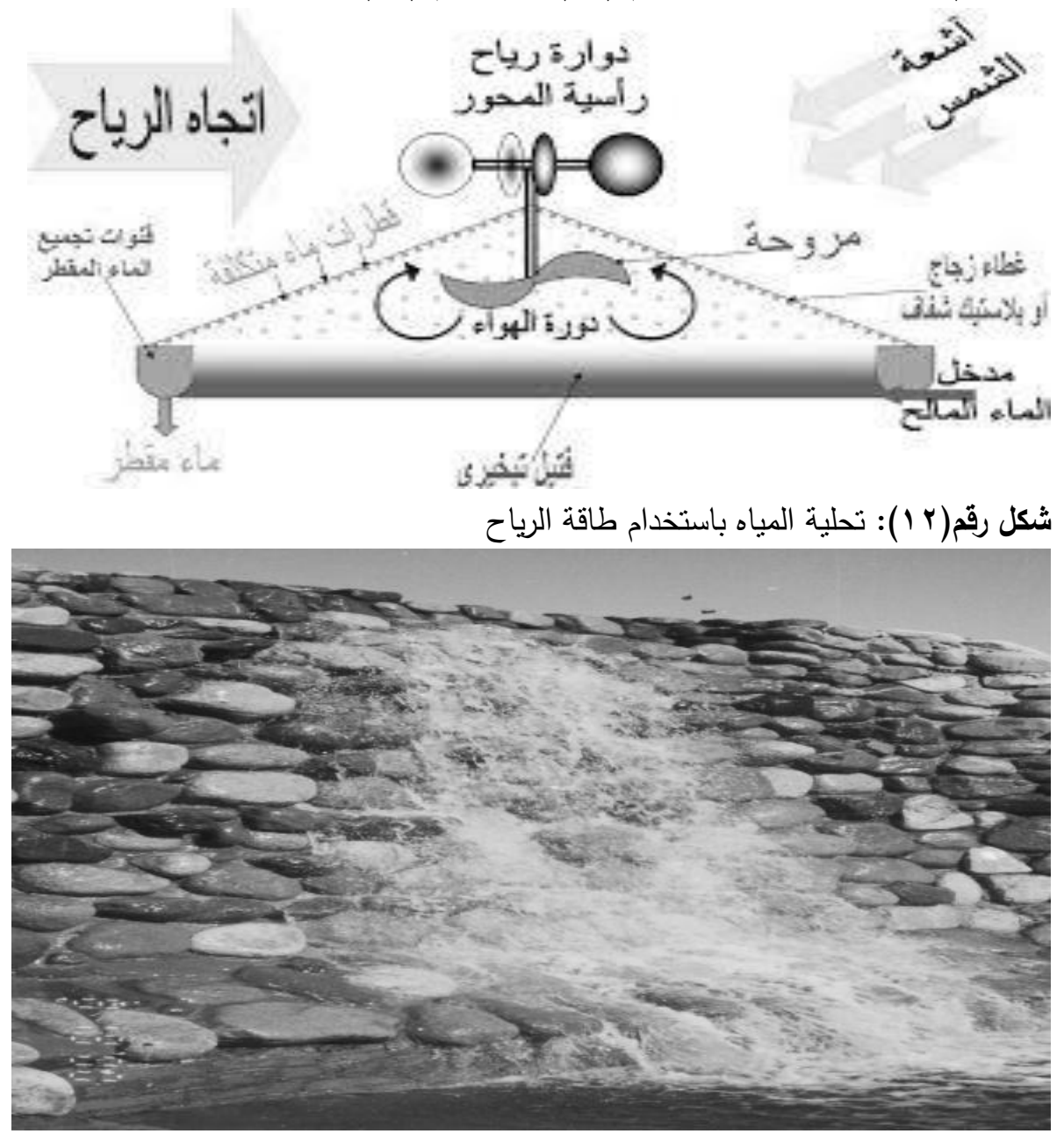

شكل رقم(ب ا ): السلسبيل (مدرج المياه "الثـلال") 


\section{الحلامية}

يتتاول البحث التصور المقترح من خلال استعراض عدد عشر معالجات هندسية تمثل فى مجماها مسطرة قياس للمسكن البيئى ذاتى الطاقة فى البيئة السيناوية وتعد أساساً مستقبلياً

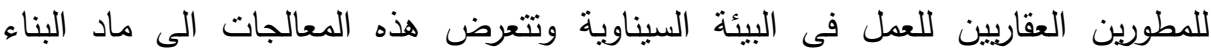
والتثطيب الداخلى والخارجى واسلوب تعظيم الاستفادة من الإضاءة والتهوية الطبيعية وايجاد مصادر طاقة متجددة داخل المسكن بالاضافة الى ايجاد طرق بيئية وحيوية للتخلص من التئية النفايات العضوية. ويستعرض انثاء نموذج تطبيقى للمعالجات الواردة بمسطرة القياس وذللك الكاد بمنطقة حمام موسى بمدينة طور سيناء فيتعرض البحث بالثرح لطبيعة الدكان وناريخه واهيته البيئية والعناصر العرانية الموجودة به. حيث يمكن منيك من اتباع ما ورد في مسطرة القياس ومدى تحقق عناصرها فى التصميم البيئى للمساكن تحديد درجة تقييم للتصميم المقترح

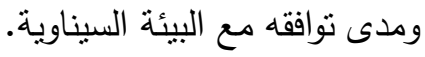

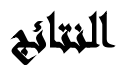

1- اعداد مسطرة قياس مكونة من عدد عشر معالجات هندسية لقياس مدى توافق التصميم مع

$$
\text { التقييم البيئى وفقا للبيئة السيناوية. }
$$

ץ- لاستفادة بمواد البناء المحلية متل الاحجار الجرانيت المقطع من الجبال بفعل الأمطار

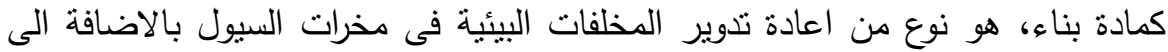

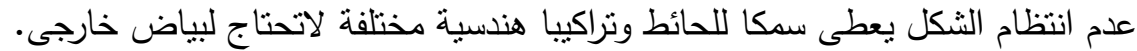
r-المونة المخلوطة بمجروش القتشريات البحرية تعمل على تقليل معامل النفاذ الحرارى اللمونة بشكل ملحوظ، وتقليل معامل النفاذ الصوتى للمونة وذلك لتعدد الطبقات التى تمر

$$
\text { علها الموجات الصوتية واختلاف درجة نفاذيتها. }
$$

ع-استخدام الاسقف الخشبية المنداخلة مع ألواح البولى كربونايت يعطى نسبة المساحة

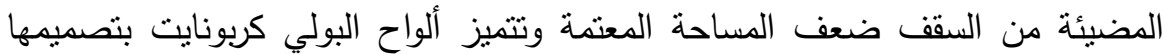

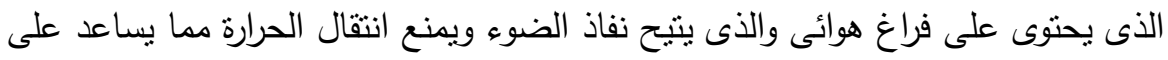
توفير بيئة صحية داخلية ويرشد من استهلاك الطاقة.

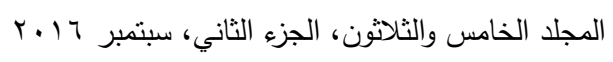


مجلة العلوم البيئية

معهد الدراسات والبحوث البيئية - جامعة عين شمس

ه-ملاقف الهواء تساعد على التخفيف من درجات الحرارة المرتفعة داخل المسكن.

\section{المئوسيامت}

1-تبنى الدولة لأهمية إستخدام الموارد الطبيعية كالطاقة الثمسية، وطاقة الرياح وطاقة البيوجاز لترشيد استهلاك الطاقة.

r-تحفيز الدولة والجهات المعنية على إستخدام مواد البناء المتاحة طبيعيا مما يعطى شكلا معماريا مميزا للإقليم ويوفر من تكاليف الإنتاج والنقل وبالتالى يوفر من التكلفة الإجمالية للمساكن.

r-الإلتزام بالمعدلات والمعايير التخطيطية والاشتراطات البيئية والتصميمية التى تتلائم مع البيئة السكنية والمناخية المتقردة لمجتمع سيناء.

\section{entl}

محمد على(0 10 + ( ): العمارة والبيئة، مكتبة مدبولى.

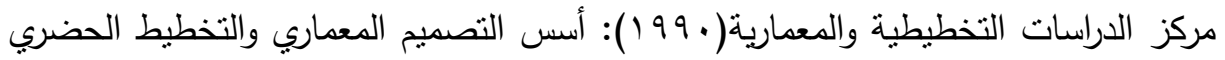

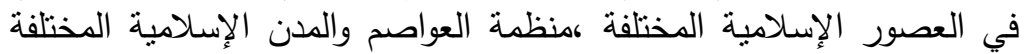
بالعاصمة القاهرة كمنظمة العواصم والمدن الإسلامية ،جدة.

وزارة الاسكان والمرافق والتتمية العمرانية(؟ . . ץ): الكود المصرى لتحسين كفاءة إستهلاك الطاقة فى المبانى السكنية. وزارة الاسكان والمرافق والتتمية العمرانية( • • ب): الكود المصرى للتهوية فى المبانى. Energy Concervation Building Code ، (2008): Ministry of PowerGovernment of India .

Energy Efficient Building Code User Guide ،(2009): Government of India . 
ماجدة إكرام عبيد وآخرون

\title{
SUGGESTED PROTOTYPE FOR AN ECOLOGICAL HOUSING MATCHED SINAI LOCAL COMMUNITY NEEDS MOSSES POOL - TUR SINAI
}

Ebeid, Magda, E. ${ }^{(1)}$; Hassanen, M. M. ${ }^{(2)}$ and Farid, M. W. ${ }^{(3)}$

1) Institute of Environmental Studies and Research, Ain Shams Universty 2) Tourism Development Authority 3) Moses pool, Tur Sinai

\begin{abstract}
Experiencing housing in Sinai Bedouin settlement areas and in particular for example Moses bath area Sinai from multiple problems and perhaps one of the most important problems facing desert development planners and especially in Sinai sense of alienation among the beneficiary population of those houses as a result from the use of local building materials, and the incompatibility of Central Government designs with the Sinai local community privacy requirements and cultural heritage of the local people of nomadic origins. That's why the idea of ecological dwelling building achieves environmental requirements Sinai may help to provide adequate housing for Bedouins in the Sinai and the synagogues as well as ecological housing for Sinai becomes a strong contender in the exploitation of the environment and its vocabulary in architectural design contributes to save energy wasted in the construction materials industry as well as in transport, as the different architectural and urban features Sinai, as well as the environment Sinai impose conditions on housing design and chitchat in the research design using raw Sinai environment. Where we turn the importance of housing and Bedouin need to provide basic requirements when you design, with a focus on the importance of using local and environmental resources that provide the needs of this community. Search and review new experience in providing housing fulfills the
\end{abstract}

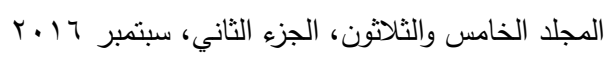


مجلة العلوم البيئية

معهد الدراسات والبحوث البيئية - جامعة عين شمس له

requirements of Bedouin make modern technology in saving and rationalizing energy inside the apartment building.

Key words: Sinai, Bedouin, Ecological. 